\title{
Epidemic Peak for COVID-19 in India, 2020
}

\author{
Chaitanya S. Wagh ${ }^{1}$ \\ Department of Computer Engg. \\ SKN College of Engineering, Pune \\ cwagh12@gmail.com
}

\author{
Parikshit N. Mahalle ${ }^{2}$ \\ Department of Computer Engg. \\ SKN College of Engineering, Pune \\ aalborg.pnm@gmail.com
}

\author{
Sanjeev J. Wagh $^{3}$ \\ Department of IT/CSE \\ Govt. College of Engineering, Karad \\ sanjeev.wagh@gcekarad.ac.in
}

\begin{abstract}
:
In India the first case of coronavirus disease 2019 (COVID-19) reported on 30 January 2020, and thereafter cases were increasing daily after the last week of Feb. 2020. COVID-19 identified as family member of coronaviridae where previously Middle East Respiratory Syndrome MERS and Severe Acute Respiratory Syndrome SARS belongs to same family. The COVID-19 attacks on respiratory system signing fever, cough and breath shortness, in severe cases may cause pneumonia, SARS or some time death.

The aim of this study work is to develop model which predicts the epidemic peak for COVID-19 in India by using the real-time data from 30 Jan to 10 May 2020. There are uncertainties while identifying the population information due to the incomplete and inaccurate data, we initiate the most popular model for epidemic prediction i.e Susceptible, Exposed, Infectious, \& Recovered SEIR initially the compartmental model for the prediction. Based on the solution of the state estimation problem for polynomial system with Poisson noise, we estimate that the epidemic peak may reach the early-middle July 2020, initializing recovered R0 to 0 and Infected I0 to 1 . The outcomes of the model will help epidemiologist to isolate the source of the disease geospatially and analyze the death. Also government authorities will be able to target their interventions for rapidly checking the spread of the epidemic.
\end{abstract}

Keywords: COVID-19, epidemic diseases, compartmental model, prediction, etc

\section{Introduction}

Due to coronavirus spread, 212 countries and territories were affected around the world with 2 majorly populated cruses: the Diamond Princess harbored in Yokohama, Japan, and the Holland America's $M S$ Zaandam cruise ship till end of April 2020. Due to outbreak of COVID-19, the unavoidable situation created where authorities of various countries, continents have to put restrictions on movement of people and non-essential activities i.e. lockdowns, maintaining social distances, work from home in academics and business continuity plans. Also the coronavirus has changed the lifestyle of human being, for working, playing and learning. Almost schools were shut, sports leagues have been canceled, and motivated to work from home using various technical platform including financial transactions.

The stalwarts in Artificial Intelligence have now started to find the solution have to help mankind in this pandemic situation using technical skills. The skills to forecast, predicts the future situation globally using various Machine learning, Deep learning techniques. Also providing alternatives to sustain the academics, business and maintained financial situations is another objective. Coronavirus prediction in Machine learning is predicting coronavirus and spread of the virus based on the affected corona patients' data [2]. In countries like Spain and Italy, doctors have to choose patients who have to be treated first because of the increase in coronavirus patients. Machine learning helps in predicting who has to be treated first from inferences made on CT scans and X rays of patients [3]. Machine learning can be used to forecast the spread of coronavirus so that doctors and the government take preventive measures. 
There are plenty of articles on information, precautionary measure and probable care list on coronavirus, also role of AI for same. Some of the studies explore comprehensive view about AI for coronavirus measures. In this paper we considered cases from India with relative datasets and suitable modeling with machine learning applied to coronavirus. The data analysis using machine learning hopes to accelerate the findings and give further direction to fight the situation to minimize the effects of the coronavirus in colligation with planning and global directions [16].

\section{Related Work}

The predictive model proposed using WEKA tool for prediction of confirmed, recovered and death cases [1]. The data model is designed to perform time series forecasting, using china dataset. The relative information to confirm cases per day with reference to inputs are demonstrated compared and concludes on Indian datasets by indicating for similarity. The model Susceptible-Infectious-Recovered-Dead i.e. SIRD proposed to estimate, basic reproduction $\mathrm{R}_{0}$, daily infection mortality and recovered rates [2]. As per observation, the cases with asymptomatic or mildly infection, infected individuals, and iterating the model as per inputs, the expected value of $R 0$ is 4.6 , while 3.2 for $20 \mathrm{x}$ confirmed cases and $40 \mathrm{x}$ recovered cases.

The proposal AIRMA i.e. Auto-Regressive Integrated Moving average model based on Time series demonstrated the prediction of most affected countries in Europe i.e. Italy Spain and France. The datasets based on world health organization provided to several variant of AIRMA models and results to identification of trends of COVID-19 and cleared the epidemiological stages in these countries [3]. The model proposed by Bushra et. \& 1. [4] Generalize additive Model (GAM), where three time dependent parameters are considered for estimation of corona outbreaks, i.e. transmission rate, recovery rate and death rate, fitting restricted maximum likelihood.

Another proposal from LI Siji and et. l. derive e-ISHR model which is based on basic SEIR model [5]. In addition of parameters i.e. hospital system, and time delay, settled the model in three stages - Initial natural stage, intermediate control and late control. The proposa effectively concludes spread rate of the disease in Wuhan and Cjhina except Hubei. The new data driven model for forecasting the coming 30 days cases in India about COVID-19 is explored. The parameters considered here are recovered cases, daily positive cases and death cases using Long Short Term Model [6]. Also the authors guess, the impact of virus can be reduced applying effective measures i.e. lockdown, social distancing and preventive measures.

\section{Methodology}

The stalwart in Artificial Intelligence and machine learning have started work on prediction model for the outbreak of the COVID-19 considering various parameters like early detection and intervention of health care and social acts like isolation, mitigation, segmentation, etc. Timely understanding the spreading process of transmissible disease is very important. The standard method to simulate transmissible pathogens is compartmental epidemiological models, which examine the transmission motion of contagious disease in host inhabitants. Standard epidemiological models are usually compartmental, where an inhabitant i.e. populations is divided into different compartments [8], and then using fractional calculus, predictions are made to see how these compartments will change over time.

The SEIR Compartmental epidemiological models help to simplify the mathematical modeling of contagious or infectious disease. Assuming that every individual in same compartment have same characteristics, the population is divided into 4 (S/E/I/R). 


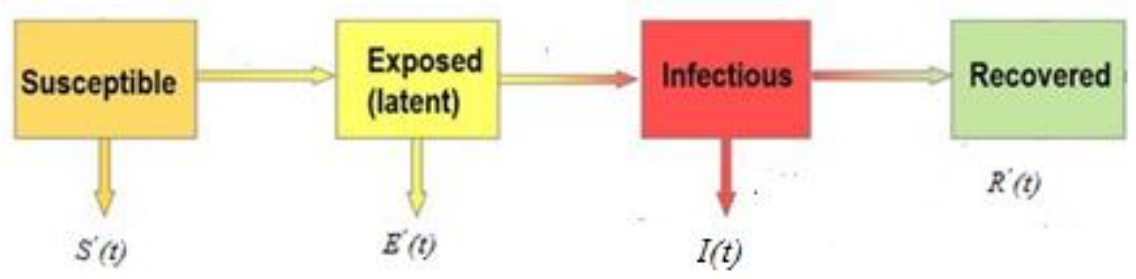

Figure 1: SEIR compartmental epidemiological model

The mathematical model underlay support the explanation of COVID-19 cases, initiate with the classic SEIR model, which groups individuals within a set population into different epidemiological classes [8]:

$S^{\prime}(t)=-\beta S(t) I(t), E^{\prime}(t)=\beta S(t) I(t)-\varepsilon E(t), I^{\prime}(t)=\varepsilon E(t)-\gamma I(t), R^{\prime}(t)=\gamma I(t), t>0$,

where $\mathrm{S}(\mathrm{t})$, denotes susceptible cases, $\mathrm{E}(\mathrm{t})$ denotes exposed cases, $\mathrm{I}(\mathrm{t})$ denotes infective cases and $\mathrm{R}(\mathrm{t})$ denote the recovered or discharged cases at time $t$, respectively. $\beta$ denotes infection rate, , $\varepsilon$ denotes onset rate and $\gamma$ denote the removal rate, respectively. $1 / \varepsilon$ is the average incubation period and $1 / \gamma$ is the average infectious period.

The significant incubation period vary from person to person with response to their immunity and during incubation may or not infectious to others. During the period of incubation the individuals are considered in the exposed stage.

The population size $N$ is

$$
\mathrm{N}=\mathrm{S}(\mathrm{t})+\mathrm{E}(\mathrm{t})+\mathrm{I}(\mathrm{t})+\mathrm{R}(\mathrm{t})
$$

Where $S$ is susceptible at time $t, E$ is exposed at time, $I$ is infective at time $t$ and $R$ is removed populations at time $t$, respectively

Assumptions: Unit time $=01$ day, $1 / \varepsilon=5$, and thus, $\varepsilon=0.2$ and $\gamma=0.1$ as per previous study [9] one infective person is identified at time $\mathrm{t}=0$ among total population

The total population can be fixed to $\mathrm{S}+\mathrm{E}+\mathrm{I}+\mathrm{R}=1, \mathrm{~N}=13.80 \times 10^{8}$ number of people in India. That is, $\gamma(0)=\operatorname{pI}(0) \times 13.80 \times 10^{8}=1$, Where $\gamma(\mathrm{t})=\mathrm{pI}(\mathrm{t}) \times 13.80 \times 10^{8}$

\section{Data Collection}

In this work, we regained the reported coronavirus positive cases (dataset source: Ministry of health and family welfare India), the number of infected population considered till $9^{\text {th }}$ May 2020 shown in Tablw 1. Daily Mohfw [13] reports were used as the number of reported infected. If any data was discovered as missing (not 0 -valued, but missing) for any day, the number of infected was determined by mohfw query (using a search for alerts for coronavirus infected in states of India). 
Table1: The reported COVID-19 cases in India until 9 May 2020 [Source MoHFW, Govt. of India].

\begin{tabular}{|c|c|c|c|c|}
\hline \begin{tabular}{|c|} 
Sr. \\
No.
\end{tabular} & Name of State / UT & $\begin{array}{c}\text { Total Confirmed } \\
\text { cases }^{\$}\end{array}$ & $\begin{array}{c}\text { Cured/Discharged/ } \\
\text { Migrated }\end{array}$ & Deaths $^{\#}$ \\
\hline 1 & Andaman and Nicobar Islands & 33 & 33 & 0 \\
\hline 2 & Andhra Pradesh & 1887 & 842 & 41 \\
\hline 3 & Arunachal Pradesh & 1 & 1 & 0 \\
\hline 4 & Assam & 59 & 34 & 1 \\
\hline 5 & Bihar & 571 & 297 & 5 \\
\hline 6 & Chandigarh & 150 & 21 & 1 \\
\hline 7 & Chhattisgarh & 59 & 38 & 0 \\
\hline 8 & Dadar Nagar Haveli & 1 & 0 & 0 \\
\hline 9 & Delhi & 6318 & 2020 & 68 \\
\hline 10 & Goa & 7 & 7 & 0 \\
\hline 11 & Gujarat & 7402 & 1872 & 449 \\
\hline 12 & Haryana & 647 & 279 & 8 \\
\hline 13 & Himachal Pradesh & 50 & 38 & 2 \\
\hline 14 & Jammu and Kashmir & 823 & 364 & 9 \\
\hline 15 & Jharkhand & 132 & 52 & 3 \\
\hline 16 & Karnataka & 753 & 376 & 30 \\
\hline 17 & Kerala & 503 & 484 & 4 \\
\hline 18 & Ladakh & 42 & 17 & 0 \\
\hline 19 & Madhya Pradesh & 3341 & 1349 & 200 \\
\hline 20 & Maharashtra & 19063 & 3470 & 731 \\
\hline 21 & Manipur & 2 & 2 & 0 \\
\hline 22 & Meghalaya & 12 & 10 & 1 \\
\hline 23 & Mizoram & 1 & 0 & 0 \\
\hline 24 & Odisha & 271 & 63 & 2 \\
\hline 25 & Puducherry & 9 & 6 & 0 \\
\hline 26 & Punjab & 1731 & 152 & 29 \\
\hline 27 & Rajasthan & 3579 & 1916 & 101 \\
\hline 28 & Tamil Nadu & 6009 & 1605 & 40 \\
\hline 29 & Telengana & 1133 & 700 & 29 \\
\hline 30 & Tripura & 118 & 2 & 0 \\
\hline 31 & Uttarakhand & 63 & 46 & 1 \\
\hline 32 & Uttar Pradesh & 3214 & 1387 & 66 \\
\hline 33 & West Bengal & 1678 & 364 & 160 \\
\hline $\begin{array}{l}\text { Tota } \\
\text { Indic }\end{array}$ & $\begin{array}{l}\text { al number of confirmed cases in } \\
\text { ia as on } 9^{\text {th }} \text { May } 2020\end{array}$ & $59662^{*}$ & 17847 & 1981 \\
\hline
\end{tabular}

\section{Model Implementation}

In public health, the standard tool to model any contagious disease is Compartmental mathematical. The model helps us to understand kinetics of infectious disease with any specific aspects. The model is simple to understand and clears interpretations, but may conclude extremist. Thus, depend on the population 
density; the proposed SEIR model represents the transmission of the disease and the death rate. For this work, we define the parameters as derived in expression 1.

The proposed compartmental model simulates the widespread of infective disease throughout India. As an epidemic breaks outs, the infected population travel scenarios were significantly dynamic, based on the geographical regions at the initial infection and its contacts spread with the various cities in India. The study gains insights, from current data driven approach on epidemics in cities of infected populations. Further we recommends for similar measures to proact the epidemic and to derive solutions for planning and managing casualties. The continuous affecting cases an epidemic model is challenging, hence our aim is to focus generalized action of epidemic spread in cities. Developing a minutely calibrated and accurate epidemic model is not possible since, the cases are not settled.

The model breaks the population units into three compartments. At time $t$ and for location I the compartments are as follows:

$S_{i}, t$ : the number of individuals susceptible to the disease.

$I_{i}, t$ : the number of individuals infected susceptible to the group.

$R_{i}, t$ : the number of individuals recovered or death.

The time $\mathrm{t}$ variable is discrete as the compartments are valued at a regular basis. In a fully susceptible population at any location $_{\mathrm{s}}$ at time $t$, an outbreak happens with probability P: Considering number of days $(\mathrm{t})=200$

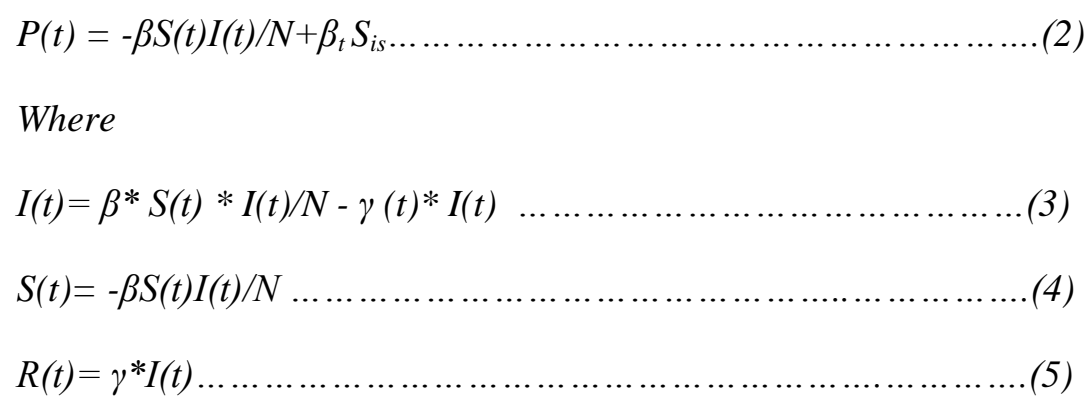

\section{Results and Discussion}

In this model we haves considered susceptibility, Infected and recovered data from the available dataset from India up to 15 April 2020, the $\beta=0.21$ (referred from WHO official website), $\gamma=$ No of days $/$ recovered $=0.1746, t=200$. 


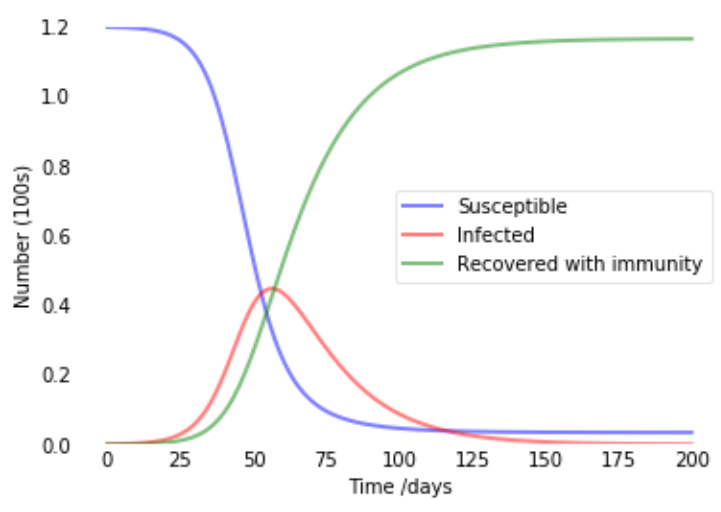

Figure 2: Current Population model

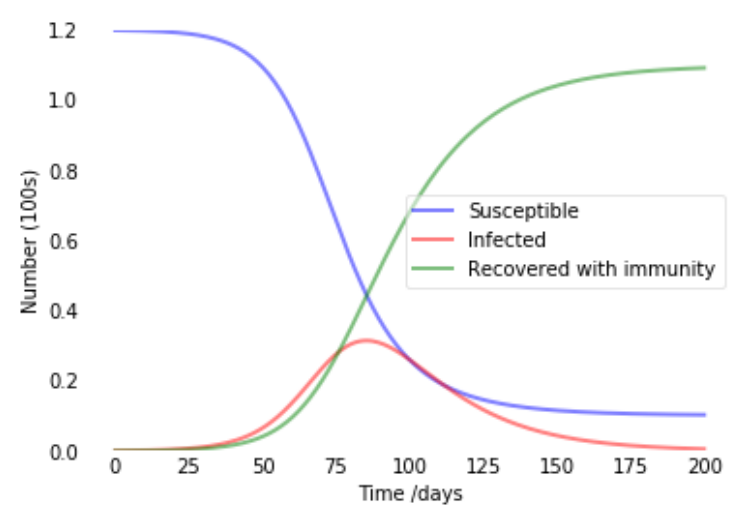

Figure 3: Predicted model after intervention of authority

The basic reproduction number $\mathrm{R}_{0}=0$ and $\mathrm{I}_{0}=1$, the epidemic peak could possibly reach the middle of July by applying the proposed SEIR compartmental model for the daily reported cases from various cities in India from 30 Jan to 09 May 2020. This result of long range peak prediction possibly contains the essential uncertainty due to the possibility of changes in the social and climatic situations. Our ultimate goal is to flatten the curve by improving policies like travel restriction, sanitization, social distancing and more testing measure. Also, the outcomes of the model indicates that the epidemic of coronavirus will not end as early in India.

The SEIR model that was used for our predictions can be easily applied to other contagious diseases with similar transmission patterns such as Whooping cough, Influenza, Ebola and Measles. Whooping cough or pertussis is a highly epidemic bacterium that lives in the mouth, nose, throat and it's life-threatening especially in infants. The widespread disease can easily disperse through coughing and sneezing of an infected individual [15]. Influenza is one of the most common contagious illnesses caused by influenza viruses. Influenza spreads simply through coughs and sneezes and can give rise to severe diseases and risk to death also from infected populations, including the elders, youngsters, childrens, and persons with chronic illnesses and weakened immune systems.

\section{Conclusion}

As earliest identification of the actual infective population, the epidemic size will be less likely to be affected. The administration and controlling authority intervention will help to flatten epidemic curve positively i.e reducing the gap between affected and recovered population. As per the situation, intervention by government authorities in support of health care units, it can be predicted to reduction of final epidemic size up to $40 \%$ which is much effective. The proposed model implemented will be also applicable to the other contagious diseases like Whooping cough, Influenza, Ebola and Measles predictions.

\section{References}

[1] Sunita Tiwari, Sushil Kumar, and Kalpna Guleria Outbreak Trends of Coronavirus Disease-2019 in India: A Prediction. DOI: https://doi.org/10.1017/dmp.2020.115, Published online by Cambridge University Press: 22 April 2020 
[2] Cleo Anastassopoulou, Lucia Russo, Athanasios Tsakris, Constantinos Siettos, “ Data-based analysis, modelling and forecasting of the COVID-19 outbreak”, Published: March 31, 2020,

https://doi.org/10.1371/journal.pone.0230405

[3] Zeynep Ceylan, "Estimation of COVID-19 Prevalence in Italy, Spain, and France”, PMID: 32360907 PMCID: PMC7175852, DOI: 10.1016/j.scitotenv.2020.138817

[4] Bushra Zareie, Amin Roshani,Mohammad Ali Mansournia, Mohammad Aziz Rasouli, Ghobad Moradi, "A Model for COVID-19 Prediction in Iran Based on China Parameters", Arch Iran Med. April 2020;23(4):244-248

[5] LI Sijia), SONG Kun, YANG Boran,GAO Yucen, GAO Xiaofeng, "Preliminary Assessment of the COVID-19 Outbreak Using3-Staged Model e-ISHR”, J. Shanghai Jiao Tong Univ. (Sci.), 2020, 25(2): 157-164 https://doi.org/10.1007/s12204-020-2169-0

[6] Anuradha Tomar, Neeraj Gupta, "Prediction for the spread of COVID-19 in India and effectiveness of preventive measures", Science of the Total Environment 728 (2020) 138762.

[7] https://www.teradata.com/Blogs/Advanced-Analytics-for-Coronavirus-Trends-Patterns-Predictions

[8] Liu, T.; Hu, J.; Kang, M.; Lin, L.; Zhong, H.; Xiao, J.; He, G.; Song, T.; Huang, Q.; Rong, Z.; et al. Transmission dynamics of 2019 novel coronavirus (2019-nCoV). bioRxive 2020.

[9] Read, J.M.; Bridgen, J.R.E.; Cummings, D.A.T.; Ho, A.; Jewell C.P. Novel coronavirus 2019-nCoV: Ealry estimation of epidemiological parameters and epidemic predictions. medRxive 2020

[10] Sun, H.; Qiu, Y.; Yan, H.; Huang, Y.; Zhu, Y.; Chen, S.X. Tracking and predicting COVID-19 epidemic inChina mainland. medRxive 2020.

[11] van den Driessche, P.; Watmough, J. Reproduction numbers and sub-threshold endemic equilibria for compartmental models of disease transmission. Math. Biosci. 2002, 180, 29-48.

[12] 12. Capaldi, A.; Behrend, S.; Berman, B.; Smith, J.; Wright, J.; Lloyd, A.L. Parameter estimation and uncertainty quantification for an epidemic model. Math. Biosci. Eng. 2012, 9, 553-576.

[13] The Japan Times. Nearly All Prefectures in Japan Shut Schools Amid Coronavirus Outbreak. Available online: https://www.japantimes.co.jp/news/2020/03/02/national/japan-prefectures-shut-schoolscovid-19/\#.XmIKE0BuKUk

[14] Ministry of Health and Family welfares, https://www.mohfw.gov.in/

[15] Kimia Ameri, and Kathryn D. Cooper, “A Network-Based Compartmental Model For The Spread Of Whooping Cough In Nebraska”, AMIA Jt Summits Transl Sci Proc. 2019; 2019: 388-397.

[16] [20] Sanjeev Wagh, Chaitanya Wagh, Parikshit Mahalle, "Epidemic Computational Model for COVID-19" Accepted for International conference on Machine Intelligence and Smart Computing, ICMISC-2020, May 20,21 2020 at GCE Karad.

[17] Gitanjali R. Shinde, Asmita B. Kalamkar, Parikshit N. Mahalle, Nilanjan Dey, Jyotismita Chaki, Aboul Ella Hassanien, "Forecasting Models for Coronavirus (COVID-19): A Survey of the State-of-theArt”, submitted Springer Nature journal Computer Science, April 2020. 